\title{
ON THE SOLUTION OF HIGHER DEGREE ALGEBRAIC EQUATIONS*
}

\author{
BY GLENN JAMES
}

1. Introduction. In this paper, we first solve for a real root of the general algebraic equation with real coefficients and negative constant term. This root appears as the limit of a function defined by a certain recursion formula. Ordinary radicals are special forms of it. By means of this result and the notation of repeated resolvent equations, we then outline a theoretically possible method of obtaining formulas for all the roots of any equation.

2. The Least Positive Real Root. Consider the equation

$$
x^{n}+a_{1} x^{n-1}+a_{2} x^{n-2}+\cdots+a_{n}=0 \text {, }
$$

where the coefficients are real and $a_{n}<0$. At least one root of this equation lies between 0 and $k$ where

$$
k>\left|a_{1}\right|+\sqrt{\left|a_{2}\right|}+\sqrt[3]{\left|a_{3}\right|}+\cdots+\sqrt[n]{\left|a_{n}\right|},
$$

and no root as large as $k . \dagger$ In order to simplify our work and our results, we subtract $k$ from the roots of (1) then make use of the interval $-k$ to 0 . The new equation is

$$
(x+k)^{n}+a_{1}(x+k)^{n-1}+\cdots+a_{n}=0 .
$$

We now denote the left member of this equation by $f(x)$ and make use of the interpolation formula

(3) $\frac{x_{p}}{x_{p-1}}=\frac{f(0)}{f(0)-f\left(x_{p-1}\right)}, \quad p=2,3, \cdots, \quad x_{1}=-k$.

* Presented to the Society, San Francisco Section, April 4, 1925.

$\dagger$ J. L. Walsh, (Annals of Mathematics, (2), vol. 25, No. 3, p. 285) proves that in the complex plane the roots all lie within or on a circle about the origin having this expression for a radius. This can be proved for real values by direct substitution. 
This takes the form*

(4) $\frac{x_{p}}{x_{p-1}}=\frac{-\left[k^{n}+a_{1} k^{n-1}+\cdots+a_{n}\right]}{x_{p-1}\left[\left(x_{p-1}+k\right)^{n-1}+\phi_{1}(k)\left(x_{p-1}+k\right)^{n-2}+\cdots+\phi_{n-1}(k)\right]}$,

where

$$
\phi_{2}(k)=k^{i}+a_{1} k^{i-1}+\cdots+a_{2} \text {. }
$$

Hence

$$
x_{p}=-\phi_{n}(k) / \theta_{n-1}\left(x_{p-1}+k\right),
$$

where

$$
\begin{aligned}
\theta_{n-1}\left(x_{p-1}+k\right)=\left(x_{p-1}+k\right)^{n-1}+\phi_{1}(k)\left(x_{p-1}+k\right)^{n-2} \\
+\cdots+\phi_{n-1}(k) .
\end{aligned}
$$

Now if the second member of (3) approaches unity as $x_{p}$ approaches some limit, say $r$, then $r$ is a root of (2), for then $\lim _{x_{p-1} \rightarrow r} f\left(x_{p-1}\right)=0$. It will suffice to show that $x_{p}$ is always negative, never decreases and does not approach zero.

The second member of (6) is negative whenever $x_{p-1} \geqq-k$, for the binomials in the denominator are then positive, the numerator is positive by virtue of our choice of $k$, and the coefficients, $\phi_{i}(k)$, are positive, since $k$ is of the form $\left|a_{1}\right|$ $+\sqrt{\left|a_{2}\right|}+\cdots \cdot+\sqrt{\left|a_{i}\right|}+c$, where $c>0$. Comparing (6) and the same relation between $x_{p+1}$ and $x_{p}$ it is easily seen that $x_{p+1} \geqq x_{p}$, provided $x_{p} \geqq x_{p-1} \geqq-k$. In the case $p=2$, the latter inequality holds. For we have $x_{1}=-k$, whence

$$
x_{2}=-\phi_{n}(k) / \phi_{n-1}(k) \text {, }
$$

and the inequality

$$
-\phi_{n}(k) / \phi_{n-1}(k) \geqq-k,
$$

reduces to $a_{n} \leqq 0$. From (6) it can be seen that $x_{p}$ cannot approach zero for it cannot exceed $-\phi_{n}(k) / \theta_{n-1}(k)$ since $x_{p-1}$ cannot be positive, and the numerator of this fraction cannot

* The denominator is most easily thrown into this form by the following device. Replace $x_{p-1}$ by $y-k$ and call the result $F(y)$. Then put $F(y)$ in the form $(y-k) Q(y)+F(k)$. Now replace $y$ by $x_{p-1}+k$ and note that $F\left(x_{p-1}+k\right)=f\left(x_{p-1}\right)$ and $F(k)=f(0)$. 
be zero since $k$ is greater than the upper limit of the roots of (1). Using (6) as a recursion formula, we can now set up the function which is a root of (2). Substituting the above value of $x_{2}$ in the second member of (6), we obtain

$$
x_{3}=-\phi_{n}(k) / \theta_{n-1}\left[k-\phi_{n}(k) / \phi_{n-1}(k)\right] ;
$$

similarly

$$
x_{4}=-\phi_{n}(k) / \theta_{n-1}\left[k-\phi_{n}(k) / \theta_{n-1}\left(k-\phi_{n}(k) / \phi_{n-1}(k)\right)\right] .
$$

The limit of this process can well be written in the form

$$
\text { . . - }-\phi_{n}(k) / \theta_{n-1}\left[k-\phi_{n}(k) / \theta_{n-1}\left(k-\phi_{n}(k) / \phi_{n-1}(k)\right)\right] \text {. }
$$

We shall denote this by $I\left[a_{1}, \cdots, a_{n}, k\right]$.

One root of equation (1) is then $I\left[a_{1}, a_{2}, \cdots, a_{n}, k\right]+k$. Obviously this is the least real, positive, root of that equation. Moreover, since by changing the sign of the roots we can make the constant term negative in an equation of odd degree, one real root of any equation except those in which the constant term is positive and the degree even takes the form

where

$$
\frac{-a_{n}}{\left|a_{n}\right|}\left[I\left(A_{1}, \cdots, A_{n}, k\right)+k\right]^{*},
$$

$$
A_{i}=\left[\frac{-a_{n}}{\left|a_{n}\right|}\right]^{i+1} a_{i} .
$$

3. The Roots of Any Equation. Suppose that, in equation (1), $n$ takes the form $2^{t} m$ where $m$ is odd. Then the degree of the resolvent equation of (1), being ${ }_{n} C_{2}$, takes the form $2^{t-1} m^{\prime}$ where $m^{\prime}$ is odd. Now forming the resolvent of the first resolvent, then the resolvent of the second, etc., we finally arrive at the $t$ th resolvent which is of odd degree. Having solved this

* It is of interest to note that, when $a_{1}=a_{2}=\cdots=a_{n-1}=0$, this is the real, $n$th root of $\left|a_{n}\right|$ if $n$ is odd and the positive, real, $n$th root of this number if $n$ is even and $a_{n}$ is negative, the rapidity of the convergence of the defining sequences depending upon our choice of $k$. 
equation for one root we are then able to find 2 roots* of the $(t-1)$ th resolvent and $2^{t}$ roots of the original equation. The procedure for finding the $m$ remaining roots is obvious.

It is a fairly simple matter to write out formulas, by this method, for the roots of equations of lower degree than the sixth, but for higher degree equations the work becomes extremely complicated.

University of California, Southern Branch

\title{
THE CONDITIONS FOR A FIXED POINT IN PROJECTIVE DIFFERENTIAL GEOMETRY
}

\author{
BY A. L. NELSON
}

1. Introduction. In the projective differential geometry of Wilczynski, as applied to various special theories, a local frame of reference is found to be useful. When theorems $\dagger$ which involve fixed points are proved by Wilczynski's methods, the conditions satisfied by the coordinates of such points, referred to such local frames, are naturally of importance. It is a conspicuous fact that these conditions invariably involve the adjoint system $\ddagger$ of differential equations. This fact the present paper undertakes to explain.

* This requires the solution of odd degree equations only. See $O n$ the solution of algebraic equations with rational coefficients, AMERICAN MATHEmatical Monthly, June, 1924, p. 286.

$\dagger$ See A. F. Carpenter, Some fundamental relations in the projective differential geometry of ruled surfaces, ANNALI DI MATEMATICA, (3), vol. 26 (1917), pp. 285 et seq. Also A. L. Nelson, Plane nets with equal invariants, RenDiconti di Palermo, vol. 41 (1916), pp. 251 et seq.

$¥$ More precisely, geometric adjoint system, in the language of Green (cf. Memoir on the general theory of surfaces and rectilinear congruences, TRANSACtions of this Society, vol. 20 (1919), p. 106). This will be further discussed in $\$ 2$.

The term "system of differential equations" will be understood in this paper to mean "completely integrable system of partial differential equations," and to include the system of one or more ordinary differential equations as a special case. 